\title{
Ghost and gluon propagators of quenched lattice QCD: NSPT versus Monte Carlo results
}

\author{
Ernst-Michael Ilgenfritz ${ }^{* \dagger}$ \\ Institut für Physik, Humboldt Universität zu Berlin \\ E-mail: ilgenfri@physik.hu-berlin.de
}

\begin{abstract}
Recent progress evaluating the Landau gauge ghost and gluon propagators within Numerical Stochastic Perturbation Theory (NSPT) has been reported at the workshop. In order to enable a confrontation with fully non-perturbative Monte Carlo calculations, the latter had to be redone based on the logarithmic definition of the gluon field in terms of the links. Experiences with the modified gauge fixing and the relation to the previous Monte Carlo results using the linear definition have been reviewed before the comparison with NSPT results was presented. Finally, the NSPT results for the two propagators up to the three-loop level as functions of $\log (a p)^{2}$ have been discussed in a form ready for comparison with future results of (diagrammatic) Lattice Perturbation Theory. Beyond the one-loop level (Kawai, Nakayama and Seo, 1981), which is nicely reproduced, the results are presently only available through NSPT.
\end{abstract}

The many faces of $Q C D$

November 2-5, 2010

Gent Belgium

\footnotetext{
*Speaker.

†Thanks go to my co-authors at Berlin, Leipzig, Parma and Regensburg [21, 22, 23].
} 


\section{Introduction}

During the last years the Landau gauge (LG) propagators in Yang-Mills theory and QCD have attracted much interest. The extreme infrared behavior was believed to encode the confining features of the gluon dynamics. Despite all attempts to simulate on very large lattices, the powerlike behavior predicted by the Gribov-Zwanziger scenario and preferred by nonperturbative continuum approaches like Schwinger-Dyson Equations [SDE] and Functional Renormalization Group [FRG] (in order to guarantee an unbroken BRST symmetry) was impossible to reproduce in lattice simulations [1]. Some exception are simulations in the $\beta \rightarrow 0$ limit [2]. Today the general opinion is that the extreme infrared behavior is very sensitive to Gribov copy effects, i.e., strongly dependent on how the residual gauge freedom is fixed, specifying what actually is the Landau gauge under discussion. The latter is insufficiently specified by the transversality condition, $\partial_{\mu} A_{\mu}=0$. Mostly the "minimal Landau gauge" has been adopted, requiring to find the absolute minimum of the socalled gauge functional (see below). The impossibility to find it, with finite computational effort, has suggested e.g. to seek improvement applying the simulated annealing algorithm [3]. Other prescriptions for the nonperturbative completion of LG have been proposed which select the "best Gribov copy" directly according to properties of the gluon or ghost propagators [4, 5].

On the other hand, hadron physics does not depend crucially on the extreme infrared behavior, the dichotomous "scaling" or "decoupling" form of the propagators. A family of decoupling solutions (with a scaling limit) actually exists as solutions of the SDE [6, 7] and FRG approach, depending on the boundary condition for the ghost propagator at zero momentum. For the gauge invariant meson bound states obtained from Bethe-Salpeter equations [8] the indifference with respect to the asymptotic behavior has been convincingly demonstrated in a recent comparative study. The behavior in the momentum range above several hundreds of $\mathrm{MeV}$, however, seems to be more important, e.g. when the QCD gluon propagator is plugged into the SDE for quark propagators $[9,10,11]$ as dynamical input. Still, the gluon propagator in this momentum range is not safely predicted by the present analytical approaches because of truncation uncertainties. Hence, there is a request for precise lattice results extrapolated to the continuum and infinite-volume limits in the intermediate momentum region, both for zero and for finite temperatures. Fortunately, in this momentum range the propagators are practically free of Gribov ambiguities.

The UV asymptotics should be well understandable by comparison with perturbation theory. The lattice propagators in this range have become an interesting source for the determination of $\Lambda_{\text {MOM }}[12,13]$ and for the attempted identification of gluon condensates $[14,15]$. On the other hand, the identification of the nonperturbative enhancement of the gluon and ghost propagators in the intermediate momentum range, over the behavior predicted by plain perturbation theory is of particular interest, too. It has been put into relation to effects of instantons [16] and shown to depend on the presence of confining degrees of freedom like vortices [17, 18, 19].

A couple of years ago we have started the study of higher loop predictions for the propagators of interest by Numerical Stochastic Perturbation Theory (NSPT). This is a powerful and very general technique [20] that represents an automatized way of doing perturbation theory. A first summary of this new application, with results restricted to the ghost propagator, has been given in Ref. [21]. In two other recent papers, we have discussed also the gluon propagator [22] and compared the NSPT results with Monte Carlo results for the gluon and ghost propagators [23]. 
The interested reader should consult these papers for more information and all details. The comparison requires a reformulation of the fully nonperturbative analysis according to the logarithmic definition of the gluon fields.

\section{Landau gauge propagators in NSPT}

Compared to continuum perturbation theory, lattice perturbation theory is much more complicated. Diagrammatical calculations of the propagators at higher loops would still be hardly feasible. We have chosen NSPT instead, which is based on a set of parallel Langevin equations, each of them for the gauge link components in a certain order in the coupling constant. Langevin simulations in general for lattice gauge theory [24] have been proposed already in the eighties [25] as an alternative to usual Monte Carlo simulations. Being an adaptation of stochastic quantization, stochastic gauge-fixing was an obligatory part in the case of gauge theories. For a recent study using stochastic gauge-fixing, with the focus on questions in close context with the Landau gauge see Ref. [26]. With stochastic gauge-fixing, transversality is only approximately satified, with all consequences for gauge dependent observables. In order to study gauge-dependent quantities, the usual Monte Carlo approach uses external gauge-fixing applied to the Monte Carlo configurations until transversality is achieved with the necessary precision. ${ }^{1}$ The same can be done for the configurations produced by Langevin simulation which are subject to measurements. For the Langevin process itself, after each update step, a single iteration of the steepest descent gauge fixing algorithm (see below) can be applied to the updated configuration in order to suppress runaway zero modes. In our approach this - and the subtraction of zero modes - completes the Langevin equation written for the links

$$
\frac{\partial}{\partial t} U_{x, \mu}(t ; \eta)=\mathrm{i}\left(\nabla_{x, \mu} S_{G}[U]-\eta_{x, \mu}(t)\right) U_{x, \mu}(t ; \eta)
$$

to a process with stochastic gauge-fixing. Here $\eta$ is a Gaussian white noise. $S_{G}$ is the respective lattice gauge field action (in our case, the Wilson action), and $\nabla_{x, \mu}$ the left Lie derivative with respect to the indicated link. Since the continuous-time limit is postponed to the final simulation data, we repeat the simulations with a set of finite time step values $\varepsilon$ and perform the update according to the stochastic evolution equation in the Euler scheme

$$
U_{x, \mu}(t+\varepsilon ; \eta)=\exp \left(\mathrm{i} F_{x, \mu}[U, \eta]\right) U_{x, \mu}(t ; \eta)
$$

Here the $\eta$-dependence is made explicit, $\eta$ entering the force additively:

$$
F_{x, \mu}[U, \eta]=\varepsilon \nabla_{x, \mu} S_{G}[U]+\sqrt{\varepsilon} \eta_{x, \mu} .
$$

For perturbative applications of the Langevin equation, a decomposition of the links into terms of definite order in the coupling constant is applied and followed through all operations. This applies to the links

$$
U_{x, \mu}(t ; \eta) \rightarrow 1+\sum_{l>0} \beta^{-l / 2} U_{x, \mu}^{(l)}(t ; \eta)
$$

\footnotetext{
${ }^{1}$ As dicussed above, various options exist how to select the "best gauge copy".
} 
and similarly to the Lie-algebra valued gluon field $A=\log U$,

$$
A_{x+\frac{\hat{\mu}}{2}, \mu}(t ; \eta)=\sum_{l>0} \beta^{-l / 2} A_{x+\frac{\hat{\mu}}{2}, \mu}^{(l)}(t ; \eta),
$$

where antisymmetry to all orders in $1 / \sqrt{\beta}$ is enforced. Here also the dependence on time $t$ (a multiple of $\varepsilon$ ) is indicated.

The $n$-loop gluon propagator in momentum-space can be practically evaluated as average over time steps (or, equivalently, as average over the noise $\eta$ )

$$
\delta^{a b} D_{\mu \nu}^{(n)}(p(k))=\left\langle\sum_{l=1}^{2 n+1}\left[\widetilde{A}_{\mu}^{a,(l)}(k) \widetilde{A}_{v}^{b,(2 n+2-l)}(-k)\right]\right\rangle_{\eta} .
$$

in terms of the Fourier-transformed gluon fields $\widetilde{A}_{\mu}^{a,(l)}(k, t ; \eta)$. For the integer-valued 4-tuples $\left(k_{1}, k_{2}, k_{3}, k_{4}\right)$ appearing in the Fourier transformation the assigned lattice momenta are:

$$
\hat{p}_{\mu}\left(k_{\mu}\right)=\frac{2}{a} \sin \left(\frac{\pi k_{\mu}}{N_{\mu}}\right)=\frac{2}{a} \sin \left(\frac{a p_{\mu}}{2}\right) .
$$

The ghost propagator in momentum space,

$$
G(p(k))=\frac{1}{N_{c}^{2}-1}\left\langle\operatorname{Tr} M^{-1}(k, t: \eta)\right\rangle_{\eta}
$$

is actually the Langevin-time average of the Fourier transformed inverse $M^{-1}(k, t ; \eta)$ of the FaddeevPopov operator

$$
M_{x y}^{a b}=\left[\partial_{\mu}^{L} D_{\mu}\right]_{x y}^{a b}
$$

which contains the backward partial derivative $\partial_{\mu}^{L}$ and the covariant derivative

$$
D_{\mu}[\varphi]=\left(1+\frac{i}{2} \Phi_{\mu}(x)-\frac{1}{12}\left(\Phi_{\mu}(x)\right)^{2}-\frac{1}{720}\left(\Phi_{\mu}(x)\right)^{4}+\frac{1}{30240}\left(\Phi_{\mu}(x)\right)^{6} \ldots\right) \partial_{\mu}^{R}+i \Phi_{\mu}(x) .
$$

The latter contains, besides the forward derivative $\partial_{\mu}^{R}$, an expansion in powers of $\left[\Phi_{\mu}\right]^{b c}=-i f_{a b c} \varphi_{\mu}^{a}$, the gluon field in the adjoint representation (with $\varphi_{\mu}^{a}=i A_{\mu}^{a}$ ), which is itself expanded in powers of $1 / \sqrt{\beta}$. The perturbative expansion of $M$ is based on collecting in $M^{(l)}$ all terms coming multiplied with the $l$-th power $\beta^{-l / 2}$ of the coupling. Then the inverse in $l$-th order is

$$
\begin{aligned}
& {\left[M^{-1}\right]^{(l)}=-\left[M^{-1}\right]^{(0)} \sum_{j=0}^{l-1} M^{(l-j)}\left[M^{-1}\right]^{(j)},} \\
& {\left[M^{-1}\right]^{(0)}=\left[M^{(0)}\right]^{-1}=\Delta^{-1} .}
\end{aligned}
$$

Thus $G^{(n)}(p(k))$ is obtained from sandwiching $\left[M^{-1}\right]^{(l=2 n)}$ between plane waves for a set of 4tuples $\left(k_{1}, k_{2}, k_{3}, k_{4}\right)$, summed over colors $b$ and taking the time average.

The standard definition of the gluon field $A_{\mu}(x)$ in terms of the site-to-site transporters (the links $U_{x, \mu}$ ) in usual Lattice Perturbation Theory (LPT) and NSPT differs from the definition adopted 
in most of the fully non-perturbative calculations (i.e., Monte Carlo simulations with external gauge-fixing), where the linear definition

$$
A_{x+\frac{\mu}{2}, \mu}^{(\operatorname{lin})}=\left.\frac{1}{2 i a g_{0}}\left(U_{x, \mu}-U_{x, \mu}^{\dagger}\right)\right|_{\text {traceless }}
$$

is employed. Before one can compare Monte Carlo with NSPT, the gauge-fixing of Monte Carlo configurations must be implemented with the logarithmic definition

$$
A_{x+\frac{\mu}{2}, \mu}^{(\log )}=\frac{1}{i a g_{0}} \log \left(U_{x, \mu}\right) .
$$

Some implications of this definition have been already studied by Furui and Nakajima [27]. In a recent paper we have posed the question of universality of the gauge-fixed lattice Monte Carlo results with respect to the two definition of the gluon field [23]. It is crucial to investigate the gluon and ghost propagators in parallel, in order to realize that both are related to their counterparts by multiplicative renormalizations, that are interrelated in such a way that the running coupling is universal. One must take into consideration that the Faddeev-Popov operator is redefined together with the gauge functional (see below) corresponding to the respective definition.

\section{Gauge fixing according to the gluon field definitions}

For the linear definition the gauge functional is

$$
F_{U}^{(\operatorname{lin})}[g]=\frac{1}{4 V} \sum_{x, \mu}\left(1-\frac{1}{3} \mathfrak{R e} \operatorname{tr}^{g} U_{x, \mu}\right),
$$

while the gauge functional for the logarithmic definition (resembling the form $\int d^{4} x \operatorname{tr} A_{\mu}^{2}(x)$ for the continuum) is

$$
F_{U}^{(\log )}[g]=\frac{1}{4 V N_{c}} \sum_{x, \mu} \operatorname{tr}\left[{ }^{g} A_{x+\frac{\mu}{2}, \mu}^{(\log )} A_{x+\frac{\mu}{2}, \mu}^{(\log )}\right] .
$$

In both cases, these functionals have to be minimized under suitable gauge transformations acting on the links $U_{x, \mu} \rightarrow{ }^{g} U_{x, \mu}=g_{x} U_{x, \mu} g_{x+\hat{\mu}}^{\dagger}$. In any relative minimum the differential Landau gauge (transversality) condition

$$
\left(\sum_{\mu} \partial_{\mu} A_{\mu}\right)(x) \equiv \sum_{\mu}\left(A_{x+\frac{\hat{\mu}}{2}, \mu}-A_{x-\frac{\hat{\mu}}{2}, \mu}\right)=0,
$$

is fulfilled for the respective gluon field, Eq. (2.13) or Eq. (2.12) and not for the other. The form of the gauge functionals essentially determines the type of gauge-fixing algorithm. The bilinear form of $F_{U}^{(\text {lin) }}[g]$ in terms of the gauge transformations $g$ suggests an overrelaxation or a simulated annealing algorithm dealing with the "spin" field $g(x)$ while $F_{U}^{(\mathrm{lin})}[g]$ plays the role of the "spin Hamiltonian". The dependence of $F_{U}^{(\log )}[g]$ on $g$ is more complicated such that a steepest gradient algorithm with updates $g_{x} \rightarrow r_{x} g_{x}$ is an adequate choice. This is realized either in the local (unaccelerated) modus with

$$
r_{x}=\exp \left(-i \alpha\left(\sum_{\mu} \partial_{\mu}^{g} A_{\mu}^{(\log )}\right)(x)\right)
$$



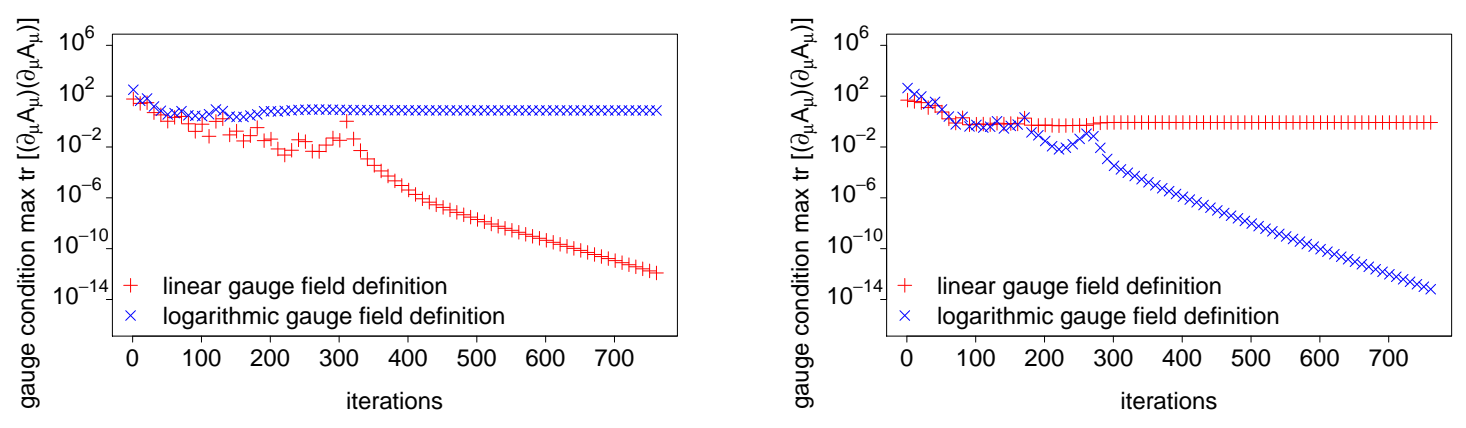

Figure 1: Change of the maximal value of the squared divergence corresponding to the two gluon field definitions during gauge fixing according to OR (linear definition, left) and the Fourier-accelerated steepest descent method (for the logarithmic definition, right) (demonstrated for a $16^{4}$ configuration at $\beta=6.0$.
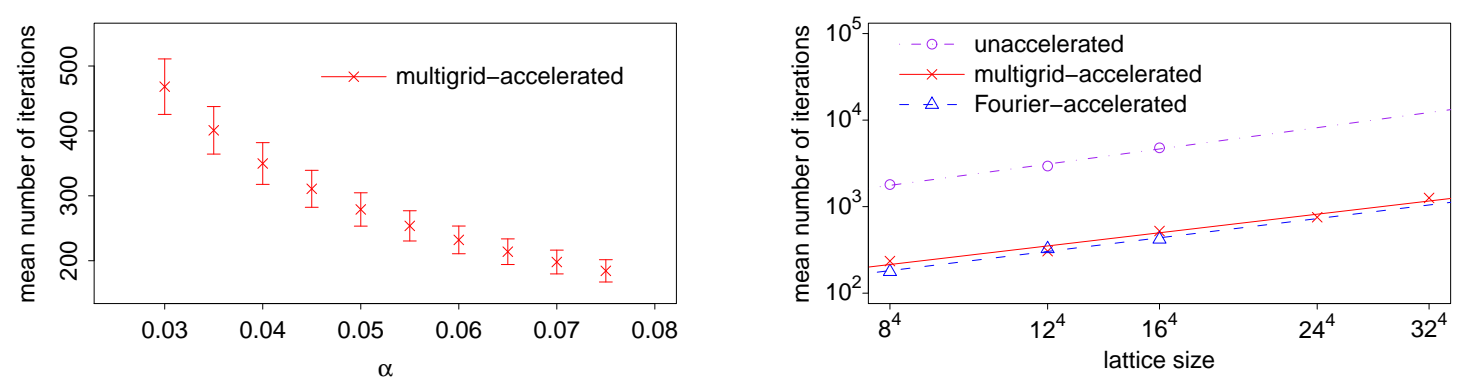

Figure 2: Number of iterations needed to reach the stopping criterion. Left: as a function of the step size parameter $\alpha$ of the multigrid-accelerated algorithm (lattice size $16^{4}, \beta=6.0$ ). Right: as a function of the lattice size for the three investigated logarithmic gauge fixing algorithms $(\beta=6.0)$.

or in a non-local, Fourier or multigrid accelerated modus

$$
r_{x}=\exp \left(-i \alpha q_{\max }^{2} \Delta^{-1}\left(\sum_{\mu} \partial_{\mu}^{g} A_{\mu}^{(\log )}\right)(x)\right) .
$$

Depending on which kind of gauge fixing is attempted, the divergence (3.3) corresponding to the complementary gauge field definition remains non-vanishing. This is demonstrated in Fig. 1. The iteration number required by some stopping criterion depends on the step-size parameter $\alpha$. It is strongly reduced when the local algorithm is replaced by the non-local, accelerated one. This is shown in Fig. 2.

The Faddeev-Popov operator has the general form

$$
M_{x y}^{a b}=A_{x}^{a b} \delta_{x y}-\sum_{\mu}\left(B_{x, \mu}^{a b} \delta_{x+\hat{\mu}, y}+C_{x, \mu}^{a b} \delta_{x-\hat{\mu}, y}\right) .
$$

For the linear definition, this is nothing but the Hessian of (3.1) with respect to infinitesimal gauge transformations $g(x)=\exp \left(i \omega^{a} T^{a}\right)$ generated by $\omega^{a}$ in the neighborhood of the gauge-fixed copy:

$$
\begin{aligned}
A_{x}^{a b} & =\mathfrak{R e t r}\left[\left\{T^{a}, T^{b}\right\} \sum_{\mu}\left(U_{x, \mu}+U_{x-\hat{\mu}, \mu}\right)\right], \\
B_{x, \mu}^{a b} & =2 \mathfrak{R e} \operatorname{tr}\left[T^{b} T^{a} U_{x, \mu}\right], \quad C_{x, \mu}^{a b}=2 \mathfrak{R e t r}\left[T^{a} T^{b} U_{x-\hat{\mu}, \mu}\right] . .
\end{aligned}
$$



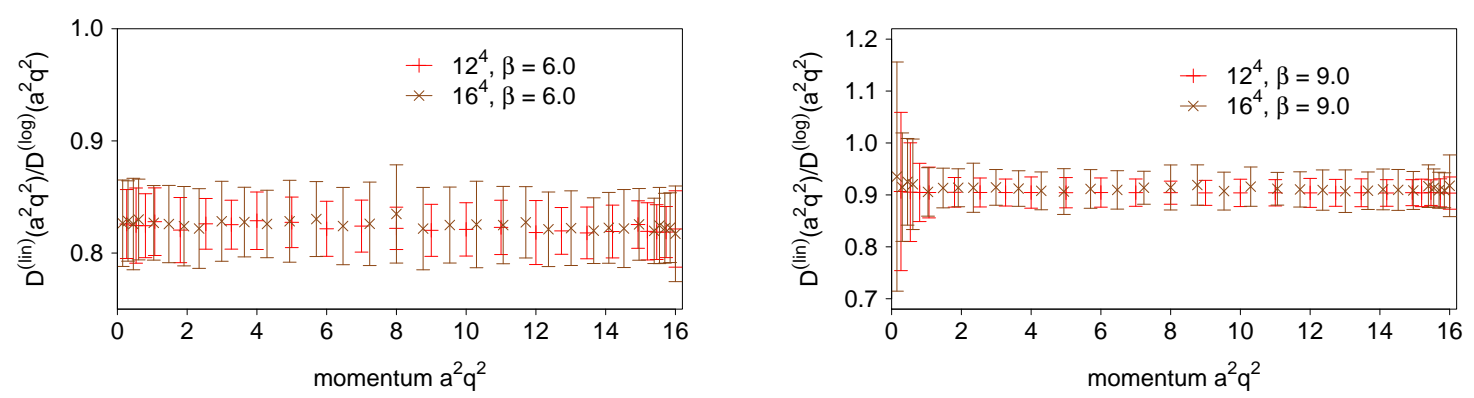

Figure 3: The ratio $C_{\text {gluon }}$ relating the gluon propagator for the two definitions of the gluon field. Data is for $\beta=6.0$ (left) and $\beta=9.0$ (right) for a $12^{4}$ and $16^{4}$ lattice.
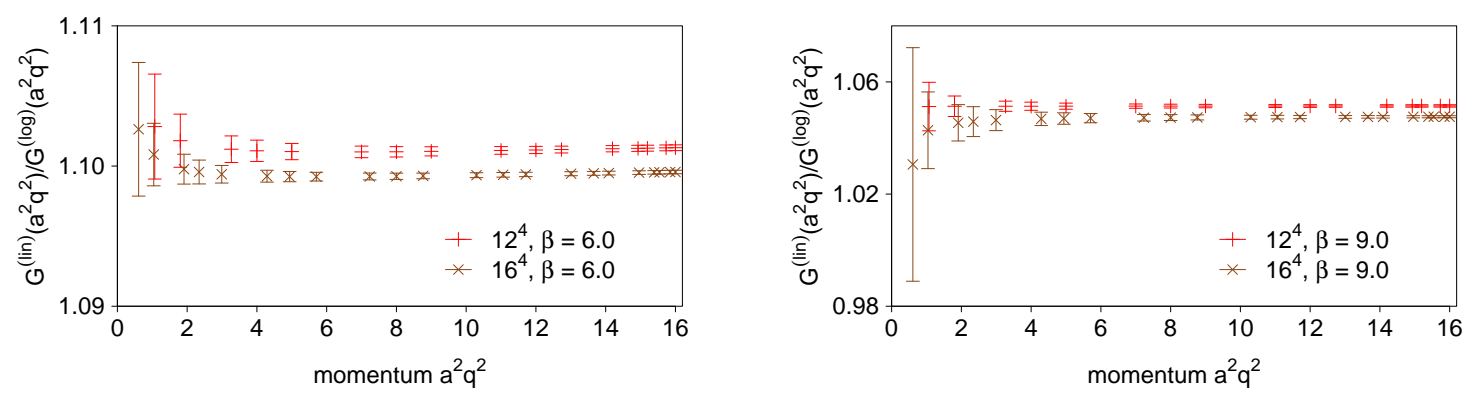

Figure 4: The ratio $C_{\text {ghost }}$ relating the ghost propagator for the two definitions of the gluon field and the respective Faddeev-Popov operator, for $12^{4}$ and $16^{4}$ lattices at $\beta=6.0$ (left) and $\beta=9.0$ (right).

For the logarithmic definition the form is:

$$
\begin{aligned}
& A_{x}^{a b}=\sum_{\mu}\left[\Omega_{x-\hat{\mu}, \mu}^{a b}+\Omega_{x, \mu}^{a b}-A_{x+\frac{\hat{\mu}}{2}, \mu}^{c} f^{a b c}\right] \\
& B_{x, \mu}^{a b}=\Omega_{x, \mu}^{a b}, \quad C_{x, \mu}^{a b}=\Omega_{x-\hat{\mu}, \mu}^{a b}-A_{x-\frac{\hat{\mu}}{2}, \mu}^{c} f^{a b c},
\end{aligned}
$$

where the closed form for $\Omega_{x, \mu}^{a b}$ gives rise to the expansion shown in (2.10). To obtain the nonperturbative ghost propagator, the full Faddeev-Popov operator is inverted with a Laplacian-preconditioned conjugate gradient algorithm and color-diagonal plane wave sources as explained in [28].

\section{Monte Carlo: linear versus logarithmic definition}

Comparing the gluon propagator and the ghost propagator for the linear definition with the respective counterpart for the logarithmic definition we found that the ratio is momentum independent within statistical errors. This is shown in Fig. 3 for the gluon propagator for two $\beta$ values and two latttice sizes. The analogous ratio for the ghost propagator at two $\beta$ values and two lattice sizes is shown in Fig. 4. The resulting renormalized gluon and ghost dressing functions for the logarithmic definition, $Z_{\mathrm{gl}}=\hat{q}^{2} D(q)$ and $Z_{\mathrm{gh}}=\hat{q}^{2} G(q)$, are generated in Fig. 5 by data points from three $\beta$ values and three corresponding lattice sizes (chosen such that the physical volume is approximately kept fixed) collapsing on a single curve. Finally, defining a renormalization group invariant running coupling through the so-called MinMom scheme [12], $\left.\alpha_{s}^{M M} q^{2}\right)=\frac{g_{0}^{2}(a)}{4 \pi} Z_{\mathrm{gl}}\left(a^{2}, q^{2}\right) Z_{\mathrm{gh}}^{2}\left(a^{2}, q^{2}\right)$, one 

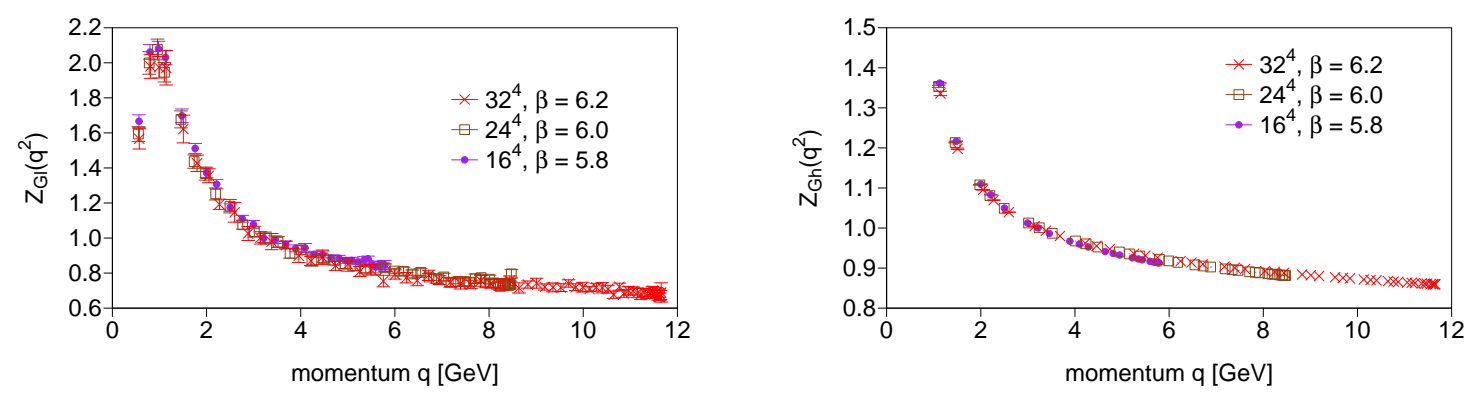

Figure 5: Renormalized gluon (left) and ghost dressing function (right) for the logarithmic definition and three various lattice spacings $a=a(\beta)$. The physical volume is thereby fixed to $V=(2.2 \mathrm{fm})^{4}$. Data has been renormalized at $q=\mu \approx 3.2 \mathrm{GeV}$.

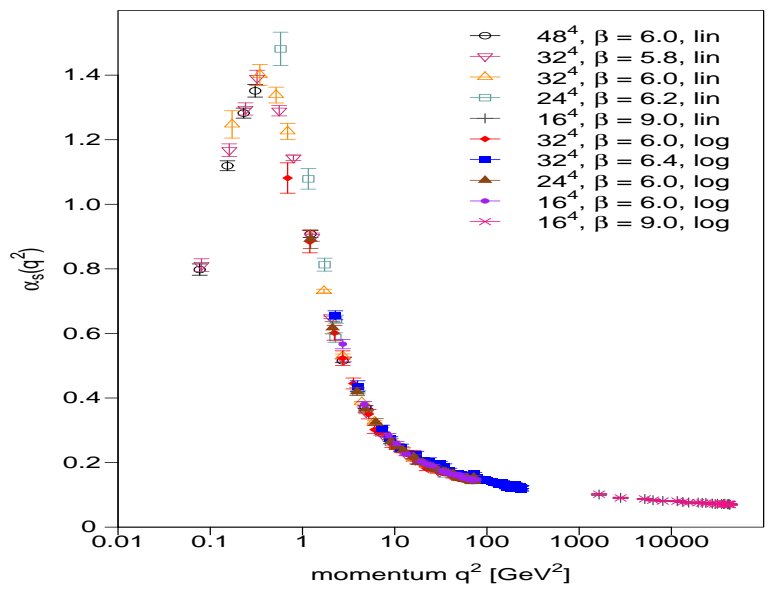

Figure 6: Running coupling for various lattice sizes and $\beta$-values. Filled symbols refer to the logarithmic definition, open symbols to the linear one.

sees data from various lattice sizes and $\beta$ values for both definitions of the gluon field falling on the single curve shown in Fig. 6.

\section{NSPT versus Monte Carlo and standard LPT}

The NSPT propagator results of $n$-loop order have to be summed up to the highest available order $n_{\max }$ by multiplying them with the appropriate powers $\beta^{-n}$. This restores the $\beta$-dependence of the propagators. We compare the fully nonperturbative results with the sum of lowest orders of NSPT in Fig. 7. The convergence can be speeded up by boosted perturbation theory, replacing $g^{2}$ as expansion parameter by $g_{b}^{2}=g^{2} / P_{\text {pert }}\left(g^{2}\right)>g^{2}$, where $P_{\text {pert }}$ is the perturbatively expanded plaquette which is always measured together with the other observables. Reordering the series in powers of $g_{b}^{2}$ gives an improved convergence. This is illustrated for the running coupling in Fig. 8 for two values of the bare coupling. The symbol $(0,0,0,0)$ means that the Monte Carlo data are restricted to the trivial joint Polyakov loop sector when the Polyakov loops in all 4 directions take almost real values. This is achieved by $Z(3)$ flipping before gauge fixing. For $\beta=9.0$ the Monte Carlo data in 

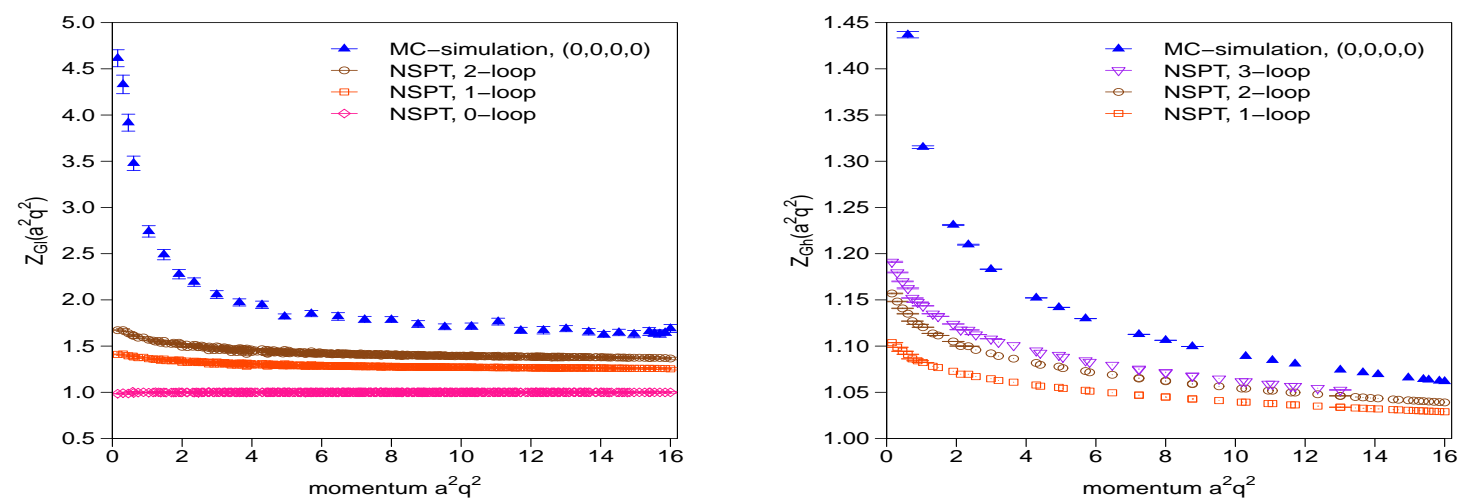

Figure 7: Comparison of MC with NSPT results for the bare gluon dressing function (left) and ghost dressing function (right) at $\beta=6.0$. The NSPT results are shown cumulatively at tree, 1-loop and 2-loop level for the gluon and at 1-loop, 2-loop and 3-loop level for the ghost.
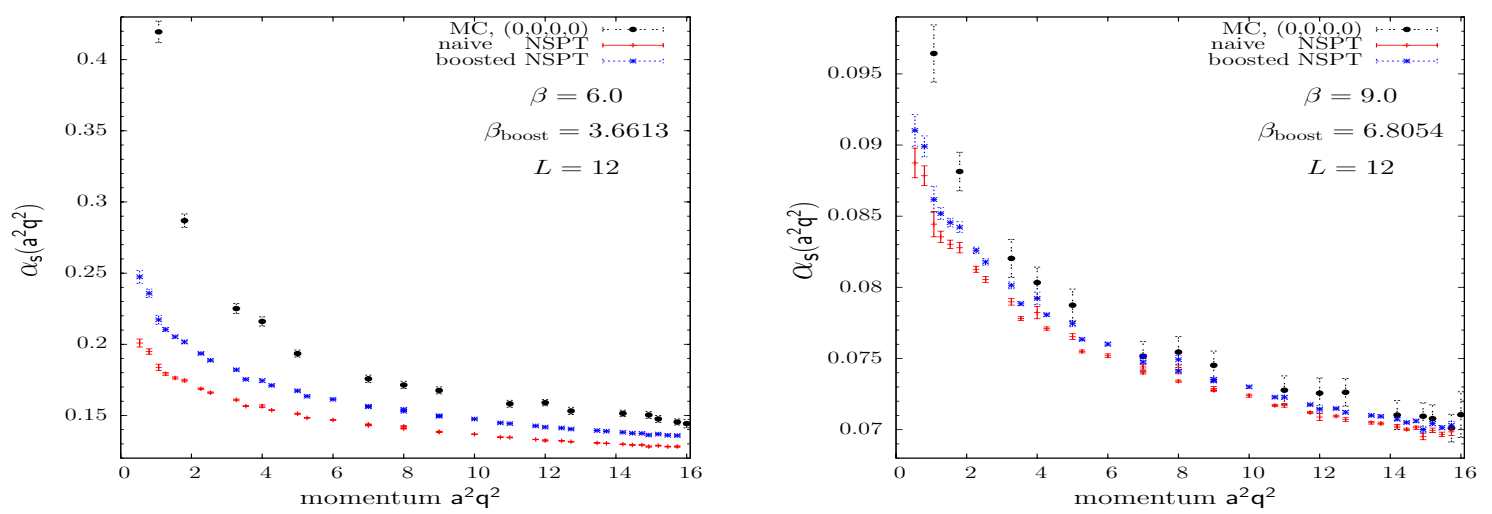

Figure 8: Comparing naive and boosted LPT (based on NSPT) data for the running coupling constant $\alpha_{s}\left(q^{2}\right)$ with corresponding MC data from the trivial Polyakov sector $(0,0,0,0)$ for a $12^{4}$ lattice. Left: $\beta=6.0$. Right: $\beta=9.0$. For the NSPT data, the gluon (ghost) dressing function up to 4-loop (3-loop) accuracy has been included.

this sector differ from measurements that leave the Polyakov loop unobserved. A convergence of NSPT to MC results in the high-momentum range is only possible for the $(0,0,0,0)$ sector.

We have developed a technique to eliminate hypercubic artefacts and finite-size effects and to perform the continuum limit. To present the results up to the three-loop level [21, 22] in the generic form (with $L=\log (p a)^{2}$ )

$$
J^{\mathrm{n}-\operatorname{loop}}(a, p, \beta)=1+\frac{1}{\beta}\left(c_{1,1} L+c_{1,0}\right)+\ldots+\frac{1}{\beta^{n}}\left(c_{n, n} L^{n}+c_{n, n-1} L^{n-1}+\ldots+c_{n, 0}\right)
$$

one needs only the leading-log terms $c_{n, n} L^{n}$ from standard LPT as external input. The non-log terms enter the non-leading-log terms of all higher loops. The one-loop results for both propagators reproduce well the analytic results of Ref. [29]. The remaining coefficients are the result of our study. 


\section{References}

[1] I. L. Bogolubsky, E.-M. Ilgenfritz, M. Müller-Preussker, and A. Sternbeck, Phys. Lett. B676, 69 (2009), arXiv:0901.0736.

[2] A. Maas, J. M. Pawlowski, D. Spielmann, A. Sternbeck, and L. von Smekal, Eur. Phys. J. C68, 183 (2010), arXiv:0912.4203.

[3] I. L. Bogolubsky, E.-M. Ilgenfritz, M. Müller-Preussker, and A. Sternbeck, PoS LAT2007, 290 (2007), arXiv:0710.1968.

[4] A. Maas, Phys. Lett. B689, 107 (2010), arXiv:0907.5185.

[5] A. Maas, PoS LATTICE2010, 279 (2010), arXiv:1010.5718.

[6] C. S Fischer, A. Maas, and J. M. Pawlowski, Annals Phys. 324, 2408 (2009), arXiv:0810.1987.

[7] C. S Fischer and L. von Smekal, arXiv:1011.6482 (2010).

[8] M. Blank, A. Krassnigg, and A. Maas, arXiv:1007.3901 (2010).

[9] C. S Fischer and R. Alkofer, Phys. Rev. D67, 094020 (2003), hep-ph/0301094.

[10] C. S Fischer and J. A. Mueller, Phys. Rev. D80, 074029 (2009), arXiv:0908.0007.

[11] C. S Fischer, A. Maas, and J. A. Mueller, Eur. Phys. J. C68, 165 (2010), arXiv:1003.1960.

[12] A. Sternbeck et al., PoS LAT2009, 210 (2009), arXiv:1003.1585.

[13] B. Blossier et al., arXiv:1012.3135 (2010).

[14] B. Blossier et al.(ETM Collaboration), Phys. Rev. D82, 034510 (2010), arXiv:1005.5290.

[15] O. Pene et al., PoS Faces of QCD, 010 (2011), arXiv:1102.1535.

[16] Ph. Boucaud et al., Phys. Rev. D70, 114503 (2004), hep-ph/0312332.

[17] K. Langfeld, H. Reinhardt, and J. Gattnar, Nucl. Phys. B621, 131 (2002), hep-ph/0107141.

[18] J. Gattnar, K. Langfeld, and H. Reinhardt, Phys. Rev. Lett. 93, 061601 (2004), hep-lat/0403011.

[19] A. Maas, Braz. J. Phys. 37, 514 (2007), hep-th/0603087.

[20] F. Di Renzo and L. Scorzato, JHEP 10, 073 (2004), hep-lat/0410010 and references therein.

[21] F. Di Renzo, E.-M. Ilgenfritz, H. Perlt, A. Schiller, and C. Torrero, Nucl. Phys. B831, 262 (2010), arXiv:0912.4152.

[22] F. Di Renzo, E.-M. Ilgenfritz, H. Perlt, A. Schiller, and C. Torrero, Nucl. Phys. B842, 122 (2011), arXiv:1008.2617.

[23] E.-M. Ilgenfritz, C. Menz, M. Müller-Preussker, A. Schiller, and A. Sternbeck, Phys. Rev. D83, 054506 (2011), arXiv:1010.5120.

[24] A. Mizutani and A. Nakamura, Nucl. Phys. Proc. Suppl. 34, 253 (1994).

[25] G. G. Batrouni et al., Phys. Rev. D32, 2736 (1985).

[26] J. M. Pawlowski, D. Spielmann, and I.-O. Stamatescu, Nucl. Phys. B830, 291 (2010), arXiv:0911.4921.

[27] S. Furui and H. Nakajima, Phys. Rev. D69, 074505 (2004), hep-lat/0305010, and references therein..

[28] A. Sternbeck, E.-M. Ilgenfritz, M. Müller-Preussker, and A. Schiller, Phys. Rev. D72, 014507 (2005), hep-lat/0506007.

[29] H. Kawai, R. Nakayama, and K. Seo, Nucl. Phys. B189, 40 (1981). 